OPEN ACCESS

Edited by:

Nadia Guerra,

Imperial College London,

United Kingdom

Reviewed by:

Adelheid Cerwenka,

Helmholtz-Gemeinschaft Deutscher

Forschungszentren (HZ), Germany

Alessandra Zingoni,

Università degli Studi di Roma La

Sapienza, Italy

*Correspondence:

Ofer Mandelboim

oferm@ekmd.huji.ac.il

Specialty section:

This article was submitted to

NK and Innate Lymphoid Cell Biology,

a section of the journal

Frontiers in Immunology

Received: 04 May 2018

Accepted: 20 August 2018

Published: 11 September 2018

Citation:

Schmiedel D and Mandelboim O (2018) NKG2D Ligands-Critical

Targets for Cancer Immune Escape and Therapy. Front. Immunol. 9:2040.

doi: 10.3389/fimmu.2018.02040

\section{NKG2D Ligands-Critical Targets for Cancer Immune Escape and Therapy}

\section{Dominik Schmiedel and Ofer Mandelboim*}

The Lautenberg Center for General and Tumor Immunology, The BioMedical Research Institute Israel Canada of the Faculty of Medicine, The Hebrew University Hadassah Medical School, Jerusalem, Israel

DNA damage, oncogene activation and excessive proliferation, chromatin modulations or oxidative stress are all important hallmarks of cancer. Interestingly, all of these abnormalities also induce a cellular stress response. By upregulating "stress-induced ligands," damaged or transformed cells can be recognized by immune cells and cleared. The human genome encodes eight functional "stress-induced ligands": MICA, MICB, and ULBP1-6. All of them are recognized by a single receptor, NKG2D, which is expressed on natural killer (NK) cells, cytotoxic T cells and other $T$ cell subsets. The NKG2D ligand/NKG2D-axis is well-recognized as an important mediator of anti-tumor activity; however, patient data about the role of NKG2D ligands in immune surveillance and escape appears conflicting. As these ligands are often actively transcribed, tumor cells are urged to manipulate the expression of these ligands on post-transcriptional or post-translational level. Although our knowledge on the regulation of NKG2D ligand expression remains fragmentary, research of the past years revealed multiple cellular mechanisms that are adopted by tumor cells to reduce the expression of "stress-induced ligands" and therefore escape immune recognition. Here, we review the post-transcriptional and post-translational mechanisms by which NKG2D ligands are modulated in cancer cells and their impact on patient prognosis. We discuss controversies and approaches to apply our understanding of the NKG2D ligand/NKG2Daxis for cancer therapy.

Keywords: NKG2D, NKG2D ligands (NKG2DL), cellular stress response, cancer therapy, immunotherapy, posttranscriptional regulation, post-translational regulation, shedding

\section{THE FAMILY OF STRESS-INDUCED LIGANDS COMPRISES HIGH DIVERSITY ON RNA AND PROTEIN LEVEL}

The NKG2D receptor is, in several aspects, an outstanding immune receptor of major interest in research and immunotherapy: First, the NKG2D receptor is expressed on lymphocytes both of the innate immune system, Natural Killer (NK) cells, as well as cytotoxic, CD4 or $\gamma \delta \mathrm{T}$ cells, which are assigned to the adaptive branch of the immune system $(1,2)$. Being considered a genuine activating receptor on NK cells, NKG2D acts as a co-stimulatory receptor on T cells $(1,3)$. Thereby, NKG2D receptor triggering induces not only cytotoxicity $(4)$, but also drives cytokine production (5-7), or impacts $\mathrm{T}$ cell differentiation and expansion $(8,9)$. Second, the NKG2D receptor recognizes eight different ligands in humans, MICA, MICB and ULBP1-6 (10). Collectively, these are termed "stress-induced ligands" since they are differentially expressed after different cellular stresses. This redundancy facilitates the immune surveillance: NKG2D alone can recognize cell suffering from infection, DNA damage, fluctuating oxygen levels, excessive 
proliferation with active tumor-promoting signaling, or heat shock. NKG2D and its ligands are therefore key proteins to mount an immune response against unhealthy cells (10). However, it must be noted that expression occurs under certain conditions also on healthy cells, especially by immune cells for immunoregulatory purposes $(11,12)$. Third, the NKG2D ligand-NKG2D axis is widely recognized as anti-tumorigenic checkpoint. NKG2D-expressing immune cells are believed to reject transforming cells prior to immune-editing, which is a prerequisite for immune escape.

However, patient data of the past years revealed conflicting data and it appears that the importance of the NKG2D receptor in tumor immune surveillance and escape is far more complex as compared to other receptor-ligand interactions we are aware of (13).

Altogether, the wide range of NKG2D-expressing immune cells, the diverse ligand repertoire and its role in cancer therapy renders this receptor as an exceptional candidate for basic and applied cancer research.

\section{DIFFERENCES AND SIMILARITIES IN THE NKG2D LIGANDS IN RNA AND PROTEIN}

The eight NKG2D ligands all belong to the family of MHC class I-like proteins. They share some degree of conservation, yet, they have distinct differences in their promoters, their RNA and protein sequences. Consequently, also their regulation is oftentimes unique and independent from each other.

As a variety of stresses induces differential expression of proteins of this family, it is not surprising that diverse cancerassociated transcription factors were previously shown to affect the expression of NKG2D ligands. Prominent examples of inducers of NKG2D ligand transcription are p53, that binds the promoter regions of ULBP1 and ULBP2 following DNA damage (14), Sp family transcription factors that influence the expression MICA, MICB and ULBP1 in proliferating cells $(15,16)$, or heat shock transcription factor 1 (HSF1) that binds its respective heat-shock elements in the promoters of MICA and MICB, respectively (17). An elaborate overview on the transcriptional regulation in humans and mice is given elsewhere (18). However, it is very clear that transcription is only the first controlled step in a multilayer of regulations that enables effective and fast induction of protein expression when required but suppresses undesired, excessive protein expression on healthy cells.

Several mechanisms how these ligands are regulated on posttranscriptional level were disclosed in the past few years that will be discussed more thoroughly below. Unsurprisingly, these important immune-modulatory molecules are complexly and mostly independently regulated and research is just on the verge of deciphering underlying regulatory networks. In the first part of this review, we will summarize known cellular, post-transcriptional mechanisms that impact NKG2D ligand expression that may be hijacked by cancer cells to evade from NKG2D-mediated surveillance. An overview on the different mechanisms is provided in Figure 1. As the biology of ULBP4
(RAET1E), ULBP5 (RAET1G), and ULBP6 (RAET1L) and their role in tumor immunity is poorly understood, we will focus on the well-studied five ligands MICA, MICB, ULBP1-3. In the second part, we will provide an overview on current approaches to use these ligands for cancer therapy which are summarized in Figure 2.

\section{REGULATORY CIRCUITS CONTROLLING NKG2D LIGAND EXPRESSION}

Due to the fact that post-transcriptional regulation mostly occurs within the untranslated regions (UTR) spanning the coding sequence upstream (5'UTR) or downstream (3'UTR) of the coding sequence, we analyzed similarities of the common variants and assessed sequence homologies using MUSCLE (19); however, we want to stress that annotations differ between databases. Also, sequences are still undergoing updates. Below, we refer to sequences found in the NCBI nuccore database (as of July 9th, 2018; NM_000247.2, NM_005931.4, NM_025218.3, NM_025217.3, NM_024518.2).

All 5'UTRs of these mRNA transcripts of all NKG2D are fairly short (below 100 nucleotides). However, the 3' UTRs, which is considered the more important site on RNA regulation, shows striking differences: MICA and MICB share 90\% homology, but about 1,000 nucleotides of the $3^{\prime} \mathrm{UTR}$ of MICB are missing in MICA. Similarly, ULBP1 and ULBP3 have extremely long UTRs of about 2,400 nucleotides, which are overall over $90 \%$ homologous. However, both UTRs contain unique regions stretching over 300 nucleotides that are not shared and therefore pose potential sites for differential regulation.

The ULBP2 3' UTR consists of only about 550 nucleotides. It contains many single nucleotide exchanges when compared to ULBP1 and ULBP3, with an overall homology of $70 \%$. Interestingly, only the beginning and the end of the sequence show conservation, whereas the sequences in between are largely missing.

Although single nucleotide exchanges exist, the observed differences suggest that the diversification in the RNA regulation following gene duplication is driven by deleterious events in the UTRs.

\section{Post-transcriptional Regulators}

MicroRNAs (miRNA or miR) were the first molecules described to impair the expression of the NKG2D ligands MICA, MICB and ULBP2 in cancer cells on mRNA level (20-24). In part, these miRNAs were shown to be overexpressed in the tumor itself ("oncomiRs"), like miR-93 that targets both MICA and MICB, but also in metastasis-associated miRNAs ("metastamiRs"), like miR-10b, that targets MICB expression (21). Interestingly, several binding sites for cellular miRNAs are overlapping with viral miRNAs (20), suggesting that the necessity to regulate stressinduced ligands using miRNA surpasses the need to evolve these sites to fight viral infections more efficiently. Next to the classical role of miRNAs to suppress protein translation, it was recently demonstrated that the 3' UTR of the stress-induced ligand MICA can switch from a short to a long version by 


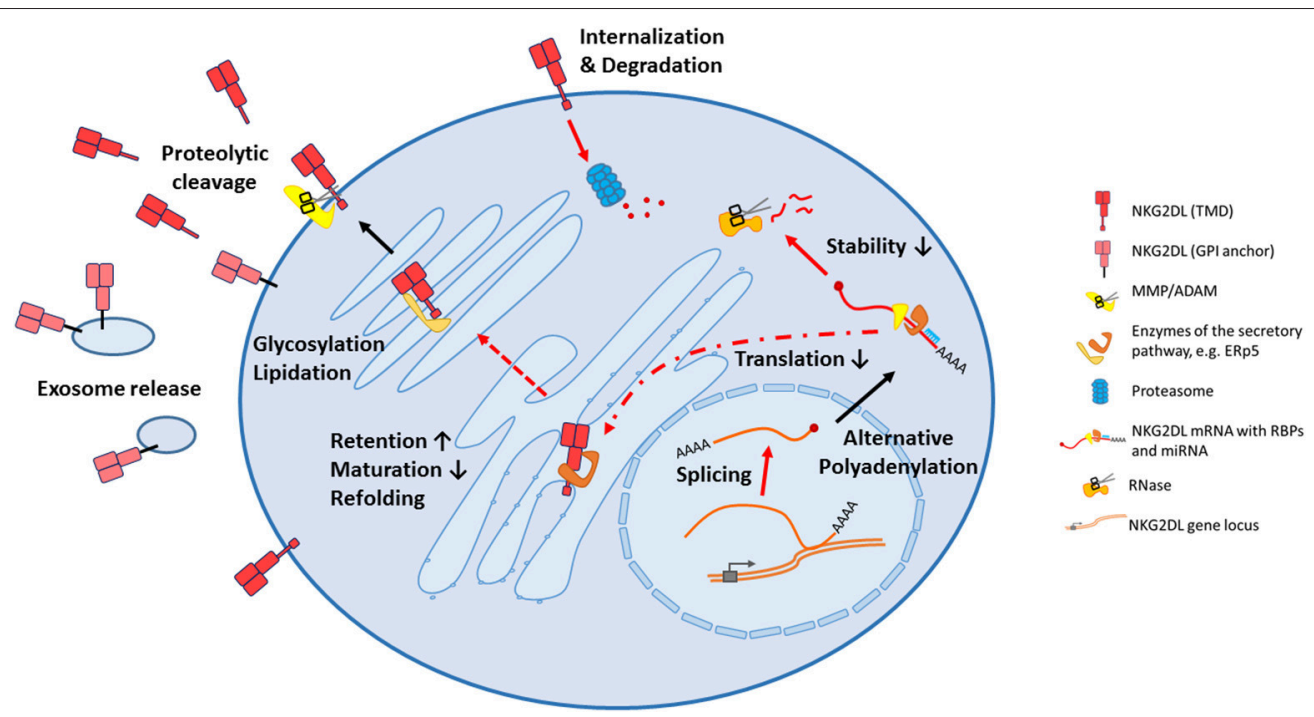

FIGURE 1 | All stages of biogenesis of NKG2D ligands can be affected in cancer cells. Following transcription, mRNA processing can be altered affecting splicing and alternative adenylation, therefore different isoforms of a ligand can be produced. After export to the cytoplasm, mRNA translation can be inhibited by miRNAs, and decay is frequently induced by RNA binding proteins. During their trafficking in endoplasmatic reticulum and golgi apparatus, the NKG2D ligands can be refolded, by instance with the help of the thioisomerase ERp5, or differentially modified by glycosylations or lipidations. Potentially, some of these modifications contribute to intracellular retention by an impaired protein maturation. Certainly, these alterations change the biological properties of these ligands once they reach the surface, with the consequence that their release from the cell surface by shedding or release in exosomes is facilitated. Alternatively, the can also be internalized and degraded by the proteasome. NKG2DL, NKG2D ligand; TMD, transmembrane domain; GPI, glycosylphosphatidylinositol; MMP, matrix metalloprotease; ADAM, a disintegrin and metalloprotease; RBP, RNA binding protein; miRNA, microRNA.

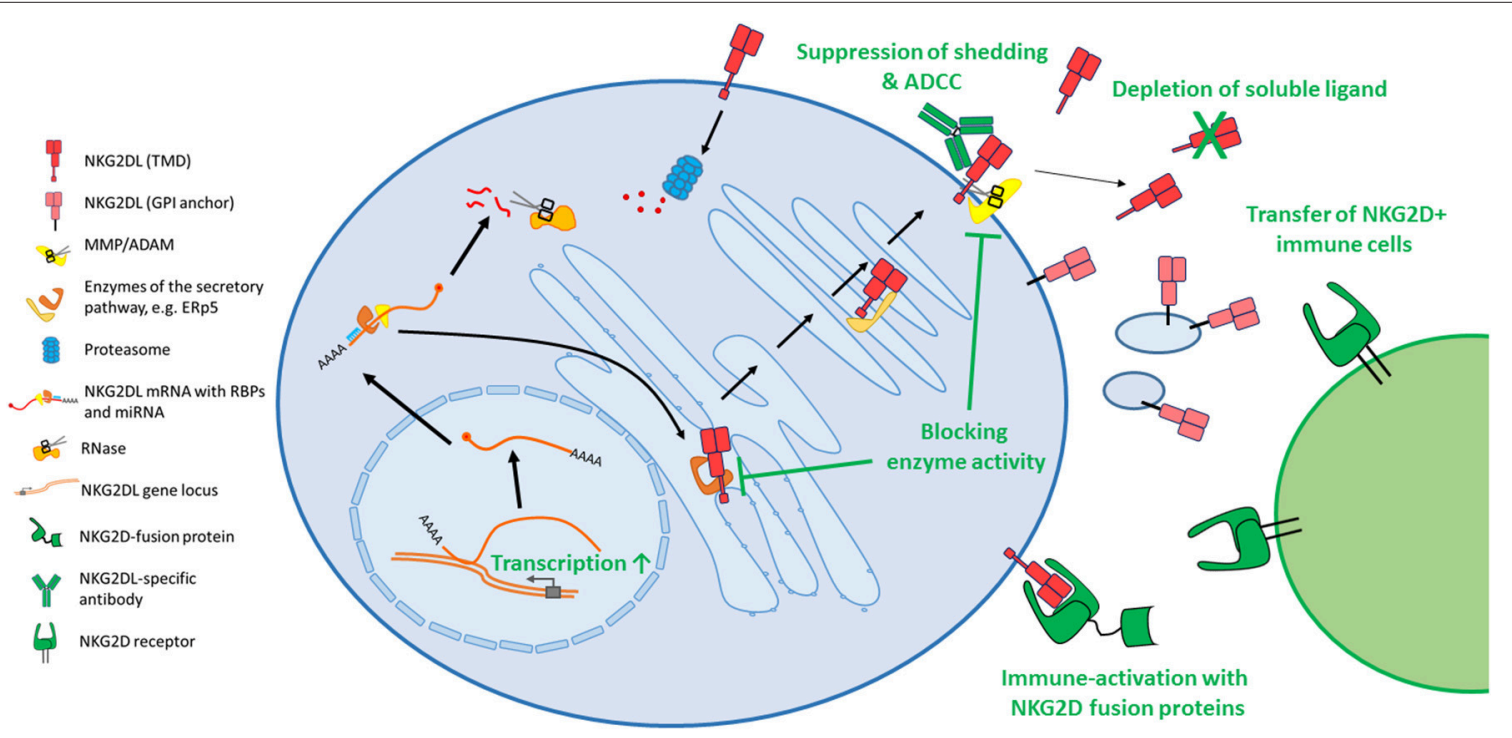

FIGURE 2 | Diverse approaches attempt to target the NKG2D axis for cancer therapy. As most cytotoxic drugs induce or constitute a cellular stress, several classes of drugs induce expression of the stress-induced NKG2D ligands, amongst DNA damaging agents, proteasome inhibitors or histone deacetylase inhibitors. In order to decrease shedding of these ligands, small molecule inhibitors targeting matrix metalloproteases were developed. Other enzymes, which are involved in protein maturation, also pose potential drug targets. Antibodies can bind the surface MICA and prevent shedding and induce ADCC. Others bind and block soluble ligands and prevent their harmful binding to immune cells. Similarly, also apheresis can reduce the load of shed ligands in serum of cancer patients. To activate the immune system toward NKG2D ligand expressing tumor cells, diverse fusion proteins were created that contain the extracellular domain of NKG2D and are linked to IL-15, anti-CD3 or an Fc portion to induce ADCC, or others. Also, the transfer of NKG2D expressing immune cells, like bone marrow grafts, donor NK cells or genetically modified T cells are approaches to fight NKG2D ligand expressing tumors. NKG2DL, NKG2D ligand; TMD, transmembrane domain; GPI, glycosylphosphatidylinositol; MMP, matrix metalloprotease; ADAM, a disintegrin and metalloprotease. 
means of alternative polyadenylation in dependence of miRNA binding (24).

What is the role of miRNAs in the regulation of NKG2D ligands in healthy conditions? One theory is, that miRNAs are capable to inhibit the translation of NKG2D ligand protein, if only little mRNA is present, for instance due to mild stresses. A minor trigger is therefore insufficient to render a healthy cell as a target for immune cell attack. According to this line of thought, miRNAs work as a "buffering capacity" and a finetuner of ligand expression that suppress ligand expression up to a certain threshold of stress (20).

Next to miRNAs, also RNA binding proteins (RBPs) were shown to interact with NKG2D ligand transcripts: MICB expression is affected by at least twelve confirmed RBPs that bind its $3^{\prime} \mathrm{UTR}$ and impact all post-transcriptional aspects like processing, turnover rate, localization or translation rate (25). Most of which suppress MICB expression and are therefore described as negative regulators. Recently, also a negative regulator binding the short 5'UTR of MICB was described (26). Interestingly, all described RBPs were shown not to affect the close relative MICA $(25,26)$. Also ULBP1 biogenesis in cancer cells is critically affected by RBPs (27), as is ULBP2 mRNA stability (28). For ULBP1 is was additionally reported that RBPs affect biogenesis of different isoforms alternative splicing (27).

Figure 1 gives a summary on the post-transcriptional regulations mentioned above. Still, pathways and critical players that affect the fate of the RNA transcripts of NKG2D ligands are only fragmentarily understood. Additionally, in many occasions, we don't know if discovered mechanisms are tumor-specific, or at least enhanced in cancer cells, or if they are simply part of the "healthy" RNA processing pathway for these ligands.

\section{Post-translational Effects}

Next to differences in the UTR regions of the ligands, major differences are also observable in the protein sequence. ULBP family members possess an $\alpha 1 / \alpha 2$ domain structure, while MIC proteins possess an $\alpha 1 / \alpha 2 / \alpha 3$ structure (29). Although being classified as a MHC class I-like protein, both families lack peptide binding ability and association with $\beta 2$-microglobulin $(18,29,30)$. On top of this layer of diversity, over 100 MIC alleles and 16 ULBP allelic variants were discovered. Notably, MICA and MICB do not possess hypervariable regions like classical MHC molecules. Genetic shuffling and point mutations occur over all three domains $(31,32)$. Another notable distinctive feature is the membrane anchorage. ULBP1, ULBP3, ULBP6 and one prevalent allele of MICA, MICA*008, are attached to the membrane by a GPI (glycosylphosphatidylinositol) anchor (33), whereas ULBP4 and ULBP5, MICB alleles and all other allelic variants of MICA are embedded into the lipid bilayer with a transmembrane domain. Uniquely, ULBP2 can be expressed both as GPI- or transmembrane anchored protein (34). Whereas, the GPI-anchored ligands localize to lipid rafts, members possessing transmembrane domain appear not to do so (35). Altogether, these proteins belong to one of the most plastic families encoded in the human genome (33).

Yet, it is not understood to what extent differences in surface localization or affinities to NKG2D give rise to differential functional outcomes when triggering the receptor. Also, we don't understand yet why the MIC proteins, and MICA in in particular, are so superior regarding their potential to create new allelic variants compared to ULBP family members.

\section{Tackling the Ligands on the Protein Level}

Like outlined above, the regulation of the eight different ligands on RNA level is multilayered and largely non-redundant. Therefore, many cancer cells can't efficiently inhibit the biogenesis of the transcripts or suppress their translation.

However, options to prevent a translated ligand from being surface-expressed exist as well. These findings are summarized in Figure 1.

First, there are several reports showing that NKG2D ligands are indeed expressed but retained intracellularly $(36,37)$. However, until now, we lack understanding which proteins are involved in this retention process that is exploited by cancer but most likely also a cellular mechanism in ligand homeostasis (37). A recent report showed that hypoxic conditions modulate MICA glycosylation and thereby prevent surface expression (38). Accordingly, glycosylation and therefore protein maturation may be one contributor in this process.

Second, surface expressed ULBP1 can be internalized and degraded by the proteasomal pathway (39). Thereby, both the levels of surface expression but also the duration of the stressresponse can be controlled.

Third, these ligands can be released from the tumor cell surface, a process termed "shedding." Cells can shed ligands either by proteolytic cleavage or by releasing ligands in exosomes.

\section{SHEDDING-AN EFFICIENT WAY TO EVADE FROM NKG2D-MEDIATED SURVEILLANCE}

Shedding constitutes a very beneficial mean for cancer cells to avoid surface expression of these ligands. Metalloproteases, most prominently ADAM10, ADAM17 (40,41), and MMP14 (42) are frequently expressed in the tumor microenvironment but also on platelets (43), cleave and thereby remove MICA, MICB, or ULBP proteins from the tumor cell surface (44-48). The process of shedding is influenced by proteins that inhibit metalloprotease activity like TIMP3 (49), or that enable or facilitate the proteolytic cleavage, like the disulfide-isomerase ERp5 (50). Accordingly, high ERp5 and ADAM10 expression were shown to yield a high load of soluble NKG2D ligands in supernatants of primary cancer cell cultures (51). Ligands, which are linked to the membrane via a GPI-anchor, like ULBP1, ULBP3 or the MICA allele $* 008$, are frequently released in exosomes $(52,53)$. For MICA, palmitoylation was shown to be crucial for co-localization with the exosome-forming protein caveolin-1 and therefore for the incorporation in exosomes (54). Although both soluble and exosomal-released NKG2D ligands bind the NKG2D receptor and mediate receptor internalization, a stronger internalization of the receptor is induced by exosomal-released ligands, probably due to their ability to crosslink the receptor on the surface $(55,56)$.

Shedding provides several major advantages for the cancer cell in terms of immune evasion. First of all, if one or several of these 
ligands are released systemically into the bloodstream of patients, they are not cell surface-exposed and therefore unable to activate NKG2D receptor-bearing cells.

More importantly, the released ligands are still capable of binding the NKG2D receptor on NK or T cells. In consequence, the NKG2D receptor is internalized in both NK and CD8 T cells (57-59). NKG2D receptor internalization is a major downside of the promiscuity of the NKG2D receptor. Whereas, a diverse array of stresses can be recognized by a functional receptor, the shedding of a single ligand is sufficient to render immune cells blind to the entire ligand family. On top, chronic engagement of the NKG2D receptor was shown to downmodulate also the activity of other NK cell receptors $(60,61)$ which may be in part connected to the degradation of the $\mathrm{CD} 3 \zeta$ signaling molecule that also impairs $\mathrm{T}$ cell activity (62). Therefore, shedding is a very powerful way to overcome NKG2D-mediated immune surveillance.

\section{NKG2D Ligands as Prognostic Marker in Cancer}

Ultimately, these differences on RNA and protein level determine NKG2D ligand regulation and expression patterns and impact thereby their importance in tumor biology.

Histological analyses of tumor samples puzzled researchers and doctors alike for several years, as the expression of NKG2D ligands was sometimes favorable and sometimes unfavorable for disease prognosis-different studies appeared contradictory $(63,64)$. However, this diversity was due to the inability to discriminate soluble and membrane expressed ligands in histology. Today we know, that solely membrane-bound ligands on cancer cells are a positive predictor for patient survival (65). However, the levels of soluble stress-induced ligands in the serum cancer patients pose a valuable prognostic factor. First, they anti-correlate with NK and T cell activity; second, they correlate to staging of the disease and have an overall negative impact on patient survival $(48,65)$. In line with the decrease in immune cell activity due to soluble NKG2D ligands, checkpoint inhibition therapy using PD-1 antibodies in melanoma was found to be most effective in absence of shed ligands (66), supporting the view that NKG2D ligands need to be taken in consideration for therapies that are not "intentionally" involve the NKG2D-axis.

\section{NKG2D Ligands-A Promising Target for Immunotherapy}

As surface-expressed NKG2D ligands promote tumor rejection and give a favorable survival prognosis, these ligands pose a promising therapeutic target for (immuno-) therapy. Diverse attempts to manipulate the expression of these ligands were undertaken in the past few years in order to harness the immune system against cancer. An overview of different strategies is given in Figure 2.

As most anti-cancer drugs act by inducing immediate cellular stress (with the ultimate goal to induce cell death), surface expression NKG2D ligands is frequently increased following treatment. Diverse compounds were identified that substantially increase stress-ligand expression and thereby render tumor cells more susceptible to immune cell attack. One prominent example is the histone deacetylase (HDAC) inhibitor valproic acid (6769) which was shown to upregulate ligands in vitro and in vivo (68). But also other drugs like hydroxyurea (70), bortezomib (71), all-trans-retinoid acid (68) or sodium butyrate $(72,73)$ appear to enhance stress-ligand expression. Therefore, patients can benefit more of some regimens if the drugs not solely damage cancer cells but also lead to the loss of immune tolerance toward the tumor $(73,74)$. However, whereas valproic acid appears to increase only the level of membrane-bound but not of soluble ligand in cell cultures $(69,75)$, other HDAC inhibitors apparently induce metalloprotease expression and might therefore also increase shedding (76). Also, an impairment of NKG2D receptor expression in NK cells upon HDAC treatment was reported $(77,78)$. Future research in this field should address this issue and assess effects on NKG2D ligands more systematically and in vivo, as frequently used in vitro models don't reflect the complexity in the interplay of tumor cells, tumor microenvironment and NKG2D expressing immune cells which are also impacted by the treatment. It is important to choose proper models that may actually predict if NKG2D mediated immune surveillance can be restored, and if patients may actually benefit of these approaches.

Whereas induction of ligands might be beneficial to activate the immune system, many late-stage cancers release ligands in soluble form and are therefore inappropriate candidates for these kind of anti-tumor strategies. The use of different inhibitors of shedding proved the concept that soluble NKG2D ligands can be effectively reduced, and that their immune-disarming properties can be reversed. Examples are inhibition of the thioreductase ERp5 (50), or prevention of proteolytic cleavage by sheddases like MMP9 (79) or ADAM10 (80). Attempts of the past years yielded selective small molecule inhibitors for MMPs like ADAM10. These are considered for cancer-therapy also for other immune-modulatory purposes besides the manipulation of NKG2D ligands which are summarized elsewhere (81). In contrast, for other potential targets, like ERp5, solely unspecific inhibitors exist at present which therefore pose no therapeutic option in the near future.

But not every new target requires a new drug development: some clinically applied drugs appear to reduce shedding of ligands as a pleasant "side effect," as shown for hypomethylating agents (49) or tyrosine kinase inhibitors (82).

However, we should bear in mind that sheddase activity is also important for the mounting immune response, for instance, the release of TNF $\alpha$ and fractalkine is mediated by $\operatorname{ADAM} 17(83,84)$.

Recently, a new antibody was developed to prevent the shedding of both MICA and MICB in order to restore NKG2D receptor activity and induce better killing of tumor cells by inducing antibody dependent cellular cytotoxicity (ADCC) (85).

However, while inhibiting shedding will be helpful in many cancer patients, exosome release of ligands is another issue that will need to be addressed. Another promising attempt uses adsorption apheresis or antibodies to reduce soluble MICA levels in the serum to restore functionality of NKG2D-bearing immune cells. In consequence, NK cell activity was successfully restored 
following depletion of soluble NKG2D ligands in plasma of cancer patients $(86,87)$.

A third, commonly exerted approach to target NKG2D ligand expressing tumor cells is the development of fusion proteins that show promising anti-tumor effects in mouse models. By using the NKG2D extracellular domain (ECD) fused to an immuneactivating component, diverse immune effector mechanisms can be targeted against NKG2D ligand expressing cells. By instance, a NKG2D receptor domain fused to the constant domain of an antibody mediates $\operatorname{ADCC}(88,89)$ via engagement of Fc receptors on immune cells. The fusion of the ECD to a single chain targeting $\mathrm{CD} 3$ directs $\mathrm{T}$ cell immune responses against the tumor cells $(90,91)$. The fusion with cytokines like IL-21 or IL-15 can activate $\mathrm{T}$ and NK cell immunity in the tumor proximity and help tumor clearance (92-94).

Last but not least, hematopoietic stem cell transplantation (HSCT) and infusions of immune cells like NK cells or genetically modified $\mathrm{T}$ cells, were also studied with particular emphasis to the function of the NKG2D receptor. HSCT is frequently exerted to treat hematopoietic malignancies $(95,96)$. Due to conditioning regimens leading to cellular stress, transplanted NKG2D expressing cells are critically involved in graft vs. leukemia but also graft vs. host disease (97). Infusions of activated NK cells to treat cancer are generally considered safe (98), and can scavenge soluble MICA in the serum of cancer patients, thereby restoring NKG2D-mediated immune surveillance (99). Also, for several years, the anti-tumor efficacy of $\mathrm{T}$ cells with possessing a transduced NKG2D chimeric antigen receptor (CAR) with different signaling domains were assessed (100). However, whereas the efficacy of these engineered cells appears to be striking in mouse models (101-103), unwanted activation and fratricide (104) of CAR T cells combined with excess cytokine release $(105,106)$ pose severe problems that likely prohibit studies in patients. The sensitivity of NKG2D CAR T cells is apparently too high so also healthy cells pose targets and lead to excess CAR T cell activation.

\section{CONCLUSIONS}

The past few years revealed a lot of new insights into the regulation of post-transcriptional and post-translational level of stress-induced ligands harboring an unforeseen complexity of regulation. We also gathered a wider understanding on how NKG2D ligands control immune responses by affecting immune cell activity, in health and disease, and opened paths how to use the NKG2D ligand axis for cancer therapy.

Yet, our understanding remains too fragmentary. Several regulatory mechanisms and factors can be attributed to determine the fate of a single ligand, but its importance for other members of the family is unknown. Certainly, our knowledge of factors affecting ligand biogenesis only scratches the surface of different layers of regulation. For instance, it appears that NKG2D ligands can be retained intracellularly, but we lack understanding how this takes place. Altogether, we miss a holistic picture, a systematic landscape of regulation, that determines which pathways (instead of single proteins or RNAs) regulating NKG2D ligands deteriorate in auto-inflammation or cancer. Such a landscape would also provide new targets, disclose new therapeutic options to harness the NKG2D axis in cancer therapy. On top, we still don't understand well, if and how different NKG2D ligands modulate immune responses differently. Can we utilize NKG2D ligands not only to kill tumor cells directly but also to orchestrate the immune response by impacting the crosstalk of immune cells? A very recent report disclosed that the NKG2D receptor triggered by ULBP2 exhibits a different nanoscale organization on the surface compared to an engagement with MICA, also leading to different functional outcomes (107), thereby giving insights to previously reported differential effects on NKG2D receptor endocytosis by binding to different ligands (108). Future studies will need to address these differences to understand the role of eight different ligands in immune homeostasis under healthy conditions as well as in cancer and autoimmunity. However, we should also bear in mind that the evolution of this diversity might be triggered by pathogens, and viruses in particular, that modulate stressinduced ligands as a mean of immune evasion (109).

Nonetheless, what we know now gives hope that the NKG2D axis might be a game changer-at least for some cancer patients. Arising methods to inhibit or deplete of soluble ligands may become more effective and easily applicable, neutralizing a strong immune inhibitor. Patients, that currently fail to mount an immune reaction when receiving checkpoint inhibitors, may regain responsiveness. Combinatorial approaches with NKG2D ligands are a very promising target to overcome the immunesuppressive tumor environment and re-activate the immune system for an anti-tumor reaction.

The next few years will show, how far we can reach out utilizing the NKG2D receptor in therapy, but we should not lose focus to advance also most basic knowledge on the NKG2D ligand and receptor axis, as every new evidence will help us to personalize (NKG2D mediated) therapies.

\section{AUTHOR CONTRIBUTIONS}

DS outlined, wrote, referenced the manuscript, and prepared the figures. OM supervised and carefully edited the work.

\section{FUNDING}

This work was supported by the German-Israeli Foundation for Scientific Research and Development (GIF) and the DKFZMOST program.

\section{ACKNOWLEDGMENTS}

We apologize to all colleagues whose work was implemented into this review due to space limitations.

In line with publishing policies of Frontiers and with the policy of the Hebrew University of Jerusalem, content which first appeared in the dissertation of DS was included in this review [Disarming cellular alarm systems-How tumors and human herpesvirus 6 alike escape immune recognition by manipulating NKG2D ligands. [Dissertation] Hebrew University of Jerusalem, Israel]. 


\section{REFERENCES}

1. Ogasawara K, Lanier LL. NKG2D in NK and T cell-mediated immunity. $J$ Clin Immunol. (2005) 25:534-40. doi: 10.1007/s10875-005-8786-4

2. Lanier LL. NKG2D in innate and adaptive immunity. Adv Exp Med Biol. (2005) 560:51-6. doi: 10.1007/0-387-24180-9_7

3. Prajapati K, Perez C, Rojas LBP, Burke B, Guevara-Patino JA. Functions of NKG2D in CD8(+) T cells: an opportunity for immunotherapy. Cell Mol Immunol. (2018) 15:470-9. doi: 10.1038/cmi.2017.161

4. Wu J, Song Y, Bakker AB, Bauer S, Spies T, Lanier LL, et al. An activating immunoreceptor complex formed by NKG2D and DAP10. Science (1999) 285:730-2.

5. Verneris MR, Karimi M, Baker J, Jayaswal A, Negrin RS. Role of NKG2D signaling in the cytotoxicity of activated and expanded $\mathrm{CD}^{+}{ }^{+} \mathrm{T}$ cells. Blood (2004) 103:3065-72. doi: 10.1182/blood-2003-06-2125

6. Cerboni C, Ardolino M, Santoni A, Zingoni A. Detuning $\mathrm{CD}^{+} \mathrm{T}$ lymphocytes by down-regulation of the activating receptor NKG2D: role of NKG2D ligands released by activated T cells. Blood (2009) 113:2955-64. doi: 10.1182/blood-2008-06-165944

7. Sheppard S, Guedes J, Mroz A, Zavitsanou AM, Kudo H, Rothery $\mathrm{SM}$, et al. The immunoreceptor NKG2D promotes tumour growth in a model of hepatocellular carcinoma. Nat Commun. (2017) 8:13930. doi: $10.1038 /$ ncomms 13930

8. Groh V, Smythe K, Dai Z, Spies T. Fas-ligand-mediated paracrine T cell regulation by the receptor NKG2D in tumor immunity. Nat Immunol. (2006) 7:755-62. doi: 10.1038/ni1350

9. Zloza A, Kohlhapp FJ, Lyons GE, Schenkel JM, Moore TV, Lacek AT, et al. NKG2D signaling on CD8(+) T cells represses T-bet and rescues CD4unhelped CD8(+) T cell memory recall but not effector responses. Nat Med. (2012) 18:422-8. doi: 10.1038/nm.2683

10. Raulet DH, Marcus A, Coscoy L. Dysregulated cellular functions and cell stress pathways provide critical cues for activating and targeting natural killer cells to transformed and infected cells. Immunol Rev. (2017) 280:93-101. doi: $10.1111 /$ imr. 12600

11. Eagle RA, Jafferji I, Barrow AD. Beyond stressed self: evidence for NKG2D ligand expression on healthy cells. Curr Immunol Rev. (2009) 5:22-34. doi: $10.2174 / 157339509787314369$

12. Trembath AP, Markiewicz MA. More than decoration: roles for natural killer group 2 member D ligand expression by immune cells. Front Immunol. (2018) 9:231. doi: 10.3389/fimmu.2018. 00231

13. Zhang J, Basher F, Wu JD. NKG2D ligands in tumor immunity: two sides of a coin. Front Immunol. (2015) 6:97. doi: 10.3389/fimmu.2015.00097

14. Textor S, Fiegler N, Arnold A, Porgador A, Hofmann TG, Cerwenka A. Human NK cells are alerted to induction of p53 in cancer cells by upregulation of the NKG2D ligands ULBP1 and ULBP2. Cancer Res. (2011) 71:5998-6009. doi: 10.1158/0008-5472.CAN-10-3211

15. Lopez-Soto A, Quinones-Lombrana A, Lopez-Arbesu R, LopezLarrea C, Gonzalez S. Transcriptional regulation of ULBP1, a human ligand of the NKG2D receptor. J Biol Chem. (2006) 281:30419-30. doi: 10.1074/jbc.M604868200

16. Rodriguez-Rodero S, Gonzalez S, Rodrigo L, Fernandez-Morera JL, Martinez-Borra J, Lopez-Vazquez A, et al. Transcriptional regulation of MICA and MICB: a novel polymorphism in MICB promoter alters transcriptional regulation by Sp1. Eur J Immunol. (2007) 37:1938-53. doi: 10.1002/eji.200737031

17. Zhang C, Wang Y, Zhou Z, Zhang J, Tian Z. Sodium butyrate upregulates expression of NKG2D ligand MICA/B in HeLa and HepG2 cell lines and increases their susceptibility to NK lysis. Cancer Immunol Immunother. (2009) 58:1275-85. doi: 10.1007/s00262-008-0645-8

18. Raulet DH, Gasser S, Gowen BG, Deng W, Jung H. Regulation of ligands for the NKG2D activating receptor. Annu Rev Immunol. (2013) 31:413-41. doi: 10.1146/annurev-immunol-032712-095951

19. Edgar RC. MUSCLE: multiple sequence alignment with high accuracy and high throughput. Nucleic Acids Res. (2004) 32:1792-7. doi: 10.1093/nar/gkh340

20. Stern-Ginossar N, Gur C, Biton M, Horwitz E, Elboim M, Stanietsky N, et al. Human microRNAs regulate stress-induced immune responses mediated by the receptor NKG2D. Nat Immunol. (2008) 9:1065-73. doi: 10.1038/ ni. 1642

21. Tsukerman P, Stern-Ginossar N, Gur C, Glasner A, Nachmani D, Bauman Y, et al. MiR-10b downregulates the stress-induced cell surface molecule MICB, a critical ligand for cancer cell recognition by natural killer cells. Cancer Res. (2012) 72:5463-72. doi: 10.1158/0008-5472.CAN-11-2671

22. Heinemann A, Zhao F, Pechlivanis S, Eberle J, Steinle A, Diederichs S, et al. Tumor suppressive microRNAs miR-34a/c control cancer cell expression of ULBP2, a stress-induced ligand of the natural killer cell receptor NKG2D. Cancer Res. (2012) 72:460-71. doi: 10.1158/0008-5472.CAN-11-1977

23. Xie J, Liu M, Li Y, Nie Y, Mi Q, Zhao S. Ovarian tumor-associated microRNA-20a decreases natural killer cell cytotoxicity by downregulating MICA/B expression. Cell Mol Immunol. (2014) 11:495-502. doi: 10.1038/ cmi.2014.30

24. Toledano T, Vitenshtein A, Stern-Ginossar N, Seidel E, Mandelboim O. Decay of the Stress-induced ligand MICA is controlled by the expression of an alternative $3^{\prime}$ untranslated region. J Immunol. (2018) 200:2819-25. doi: $10.4049 /$ iimmunol.1700968

25. Nachmani D, Gutschner T, Reches A, Diederichs S, Mandelboim O. RNAbinding proteins regulate the expression of the immune activating ligand MICB. Nat Commun. (2014) 5:4186. doi: 10.1038/ncomms5186

26. Berhani O, Nachmani D, Yamin R, Schmiedel D, Bar-On Y, Mandelboim $\mathrm{O}$. Vigilin regulates the expression of the stress-induced ligand MICB by interacting with its $5^{\prime}$ untranslated region. J Immunol. (2017) 198:3662-70. doi: 10.4049/jimmunol.1601589

27. Gowen BG, Chim B, Marceau CD, Greene TT, Burr P, Gonzalez JR, et al. A forward genetic screen reveals novel independent regulators of ULBP1, an activating ligand for natural killer cells. Elife (2015) 4:e08474. doi: 10.7554/eLife.08474

28. Schmiedel D, Tai J, Yamin R, Berhani O, Bauman Y, Mandelboim O. The RNA binding protein IMP3 facilitates tumor immune escape by downregulating the stress-induced ligands ULPB2 and MICB. Elife (2016) 5:e13426. doi: 10.7554/eLife.13426

29. Eagle RA, Trowsdale J. Promiscuity and the single receptor: NKG2D. Nat Rev Immunol. (2007) 7:737-44. doi: 10.1038/nri2144

30. Zwirner NW, Fernandez-Vina MA, Stastny P. MICA, a new polymorphic HLA-related antigen, is expressed mainly by keratinocytes, endothelial cells, and monocytes. Immunogenetics (1998) 47:139-48.

31. Elsner HA, Schroeder M, Blasczyk R. The nucleotide diversity of MICA and MICB suggests the effect of overdominant selection. Tissue Antigens (2001) 58:419-21. doi: 10.1034/j.1399-0039.2001.580612.x

32. Choy MK, Phipps ME. MICA polymorphism: biology and importance in immunity and disease. Trends Mol Med. (2010) 16:97-106. doi: 10.1016/j.molmed.2010.01.002

33. Fernandez-Messina L, Reyburn HT, Vales-Gomez M. Human NKG2Dligands: cell biology strategies to ensure immune recognition. Front Immunol. (2012) 3:299. doi: 10.3389/fimmu.2012.00299

34. Fernandez-Messina L, Ashiru O, Aguera-Gonzalez S, Reyburn HT, ValesGomez M. The human NKG2D ligand ULBP2 can be expressed at the cell surface with or without a GPI anchor and both forms can activate NK cells. $J$ Cell Sci. (2011) 124:321-7. doi: 10.1242/jcs.076042

35. Eleme K, Taner SB, Onfelt B, Collinson LM, McCann FE, Chalupny NJ, et al. Cell surface organization of stress-inducible proteins ULBP and MICA that stimulate human NK cells and T cells via NKG2D. J Exp Med. (2004) 199:1005-10. doi: 10.1084/jem.20032194

36. Fuertes MB, Girart MV, Molinero LL, Domaica CI, Rossi LE, Barrio $\mathrm{MM}$, et al. Intracellular retention of the NKG2D ligand MHC class I chain-related gene A in human melanomas confers immune privilege and prevents NK cell-mediated cytotoxicity. J Immunol. (2008) 180:4606-14. doi: 10.4049/jimmunol.180.7.4606

37. Ghadially H, Brown L, Lloyd C, Lewis L, Lewis A, Dillon J, et al. MHC class I chain-related protein A and B (MICA and MICB) are predominantly expressed intracellularly in tumour and normal tissue. Br J Cancer (2017) 116:1208-17. doi: 10.1038/bjc.2017.79

38. Yamada N, Kato-Kogoe N, Yamanegi K, Nishiura H, Fujihara Y, Fukunishi S, et al. Inhibition of asparagine-linked glycosylation participates in hypoxiainduced down-regulation of cell-surface MICA expression. Anticancer Res. (2018) 38:1353-9. doi: 10.21873/anticanres.12358 
39. Fernandez-Messina L, Reyburn HT, Vales-Gomez M. A short half-life of ULBP1 at the cell surface due to internalization and proteosomal degradation. Immunol Cell Biol. (2016) 94:479-85. doi: 10.1038/ icb. 2016.2

40. Waldhauer I, Goehlsdorf D, Gieseke F, Weinschenk T, Wittenbrink M, Ludwig A, et al. Tumor-associated MICA is shed by ADAM proteases. Cancer Res. (2008) 68:6368-76. doi: 10.1158/0008-5472. CAN-07-6768

41. Chitadze G, Lettau M, Bhat J, Wesch D, Steinle A, Furst D, et al. Shedding of endogenous MHC class I-related chain molecules A and B from different human tumor entities: heterogeneous involvement of the "a disintegrin and metalloproteases" 10 and 17. Int J Cancer (2013) 133:155766. doi: $10.1002 /$ ijc. 28174

42. Liu G, Atteridge CL, Wang X, Lundgren AD, Wu JD. The membrane type matrix metalloproteinase MMP14 mediates constitutive shedding of MHC class I chain-related molecule a independent of a disintegrin and metalloproteinases. J Immunol. (2010) 184:3346-50. doi: 10.4049/jimmunol.0903789

43. Maurer S, Kropp KN, Klein G, Steinle A, Haen SP, Walz JS, et al. Platelet-mediated shedding of NKG2D ligands impairs NK cell immune-surveillance of tumor cells. Oncoimmunology (2017) 7:e1364827. doi: 10.1080/2162402X.2017.1364827

44. Salih HR, Rammensee HG, Steinle A. Cutting edge: down-regulation of MICA on human tumors by proteolytic shedding. J Immunol. (2002) 169:4098-102. doi: 10.4049/jimmunol.169.8.4098

45. Salih HR, Antropius H, Gieseke F, Lutz SZ, Kanz L, Rammensee HG, et al. Functional expression and release of ligands for the activating immunoreceptor NKG2D in leukemia. Blood (2003) 102:1389-96. doi: 10.1182/blood-2003-01-0019

46. Waldhauer I, Steinle A. Proteolytic release of soluble UL16binding protein 2 from tumor cells. Cancer Res. (2006) 66:2520-26. doi: 10.1158/0008-5472.CAN-05-2520

47. Salih HR, Goehlsdorf D, Steinle A. Release of MICB molecules by tumor cells: mechanism and soluble MICB in sera of cancer patients. Hum Immunol. (2006) 67:188-95. doi: 10.1016/j.humimm.2006.02.008

48. Holdenrieder S, Stieber P, Peterfi A, Nagel D, Steinle A, Salih HR. Soluble MICB in malignant diseases: analysis of diagnostic significance and correlation with soluble MICA. Cancer Immunol Immunother. (2006) 55:1584-9. doi: 10.1007/s00262-006-0167-1

49. Raneros AB, Puras AM, Rodriguez RM, Colado E, Bernal T, Anguita E, et al. Increasing TIMP3 expression by hypomethylating agents diminishes soluble MICA, MICB and ULBP2 shedding in acute myeloid leukemia, facilitating NK cell-mediated immune recognition. Oncotarget (2017) 8:31959-76. doi: 10.18632/oncotarget.16657

50. Kaiser BK, Yim D, Chow IT, Gonzalez S, Dai Z, Mann HH, et al. Disulphideisomerase-enabled shedding of tumour-associated NKG2D ligands. Nature (2007)447:482-6.doi: 10.1038/nature05768

51. Zocchi MR, Catellani S, Canevali P, Tavella S, Garuti A, Villaggio B, et al. High ERp5/ADAM10 expression in lymph node microenvironment and impaired NKG2D ligands recognition in Hodgkin lymphomas. Blood (2012) 119:1479-89. doi: 10.1182/blood-2011-07-370841

52. Fernandez-Messina L, Ashiru O, Boutet P, Aguera-Gonzalez S, Skepper JN, Reyburn HT, et al. Differential mechanisms of shedding of the glycosylphosphatidylinositol (GPI)-anchored NKG2D ligands. J Biol Chem. (2010) 285:8543-51. doi: 10.1074/jbc.M109.045906

53. Ashiru O, Boutet $\mathrm{P}$, Fernandez-Messina L, Aguera-Gonzalez S, Skepper JN, Vales-Gomez M, et al. Natural killer cell cytotoxicity is suppressed by exposure to the human NKG2D ligand MICA*008 that is shed by tumor cells in exosomes. Cancer Res. (2010) 70:481-9. doi: 10.1158/0008-5472.CAN-09-1688

54. Aguera-Gonzalez S, Gross CC, Fernandez-Messina L, Ashiru O, Esteso G, Hang HC, et al. Palmitoylation of MICA, a ligand for NKG2D, mediates its recruitment to membrane microdomains and promotes its shedding. Eur J Immunol. (2011) 41:3667-76. doi: 10.1002/eji.2011 41645

55. Salih HR, Holdenrieder S, Steinle A. Soluble NKG2D ligands: prevalence, release, and functional impact. Front Biosci. (2008) 13:3448-56. doi: $10.2741 / 2939$
56. Chitadze G, Bhat J, Lettau M, Janssen O, Kabelitz D. Generation of soluble NKG2D ligands: proteolytic cleavage, exosome secretion and functional implications. Scand J Immunol. (2013) 78:120-9. doi: 10.1111/sji. 12072

57. Groh V, Wu J, Yee C, Spies T. Tumour-derived soluble MIC ligands impair expression of NKG2D and T-cell activation. Nature (2002) 419:734-8. doi: $10.1038 /$ nature 01112

58. Song H, Kim J, Cosman D, Choi I. Soluble ULBP suppresses natural killer cell activity via down-regulating NKG2D expression. Cell Immunol. (2006) 239:22-30. https://doi.org/10.1016/j.cellimm.2006.03.002

59. Lundholm M, Schroder M, Nagaeva O, Baranov V, Widmark A, MinchevaNilsson L, et al. Prostate tumor-derived exosomes down-regulate NKG2D expression on natural killer cells and CD8+ T cells: mechanism of immune evasion. PLoS ONE (2014) 9:e108925. doi: 10.1371/journal.pone.01 08925

60. Coudert JD, Scarpellino L, Gros F, Vivier E, Held W. Sustained NKG2D engagement induces cross-tolerance of multiple distinct NK cell activation pathways. Blood (2008) 111:3571-8. doi: 10.1182/blood-2007-07-1 00057

61. Koch C, Kim Y, Zoller T, Born C, Steinle A. Chronic NKG2D Engagement in vivo differentially impacts $\mathrm{NK}$ cell responsiveness by activating NK receptors. Front Immunol. (2017) 8:1466. doi: 10.3389/fimmu.2017. 01466

62. Hanaoka N, Jabri B, Dai Z, Ciszewski C, Stevens AM, Yee C, et al. NKG2D initiates caspase-mediated CD3zeta degradation and lymphocyte receptor impairments associated with human cancer and autoimmune disease. J Immunol. (2010) 185:5732-42. doi: 10.4049/jimmunol.10 02092

63. Watson NF, Spendlove I, Madjd Z, McGilvray R, Green AR, Ellis IO, et al. Expression of the stress-related MHC class I chain-related protein MICA is an indicator of good prognosis in colorectal cancer patients. Int J Cancer (2006) 118:1445-52. doi: 10.1002/ijc.21510

64. Madjd Z, Spendlove I, Moss R, Bevin S, Pinder SE, Watson NF, et al. Upregulation of MICA on high-grade invasive operable breast carcinoma. Cancer Immun. (2007) 7:17.

65. Zhao Y, Chen N, Yu Y, Zhou L, Niu C, Liu Y, et al. Prognostic value of MICA/B in cancers: a systematic review and meta-analysis. Oncotarget (2017) 8:96384-95. doi: 10.18632/oncotarget.21466

66. Maccalli C, Giannarelli D, Chiarucci C, Cutaia O, Giacobini G, Hendrickx W, et al. Soluble NKG2D ligands are biomarkers associated with the clinical outcome to immune checkpoint blockade therapy of metastatic melanoma patients. Oncoimmunology (2017) 6:e1323618. doi: 10.1080/2162402X.2017.1323618

67. Armeanu S, Bitzer M, Lauer UM, Venturelli S, Pathil A, Krusch M, et al. Natural killer cell-mediated lysis of hepatoma cells via specific induction of NKG2D ligands by the histone deacetylase inhibitor sodium valproate. Cancer Res. (2005) 65:6321-9. doi: 10.1158/0008-5472. CAN-04-4252

68. Poggi A, Catellani S, Garuti A, Pierri I, Gobbi M, Zocchi MR. Effective in vivo induction of NKG2D ligands in acute myeloid leukaemias by all-trans-retinoic acid or sodium valproate. Leukemia (2009) 23:641-8. doi: 10.1038/leu.2008.354

69. Yamanegi K, Yamane J, Kobayashi K, Kato-Kogoe N, Ohyama H, Nakasho $\mathrm{K}$, et al. Sodium valproate, a histone deacetylase inhibitor, augments the expression of cell-surface NKG2D ligands, MICA/B, without increasing their soluble forms to enhance susceptibility of human osteosarcoma cells to NK cell-mediated cytotoxicity. Oncol Rep. (2010) 24:1621-7. doi: 10.3892/or-00001026

70. Lu X, Ohata K, Kondo Y, Espinoza JL, Qi Z, Nakao S. Hydroxyurea upregulates NKG2D ligand expression in myeloid leukemia cells synergistically with valproic acid and potentially enhances susceptibility of leukemic cells to natural killer cell-mediated cytolysis. Cancer Sci. (2010) 101:609-15. doi: 10.1111/j.1349-7006.2009. 01439.x

71. Niu C, Jin H, Li M, Zhu S, Zhou L, Jin F, et al. Low-dose bortezomib increases the expression of NKG2D and DNAM-1 ligands and enhances induced $\mathrm{NK}$ and gammadelta T cell-mediated lysis in multiple myeloma. Oncotarget (2017) 8:5954-64. doi: 10.18632/oncotarget.13979 
72. Schmudde M, Braun A, Pende D, Sonnemann J, Klier U, Beck JF, et al. Histone deacetylase inhibitors sensitize tumour cells for cytotoxic effects of natural killer cells. Cancer Lett. (2008) 272:110-21. doi: 10.1016/j.canlet.2008.06.027

73. Lopez-Cobo S, Pieper N, Campos-Silva C, Garcia-Cuesta EM, Reyburn HT, Paschen A, et al. Impaired NK cell recognition of vemurafenibtreated melanoma cells is overcome by simultaneous application of histone deacetylase inhibitors. Oncoimmunology (2017) 7:e1392426. doi: 10.1080/2162402X.2017.1392426

74. Shi P, Yin T, Zhou F, Cui P, Gou S, Wang C. Valproic acid sensitizes pancreatic cancer cells to natural killer cell-mediated lysis by upregulating MICA and MICB via the PI3K/Akt signaling pathway. BMC Cancer (2014) 14:370. doi: 10.1186/1471-2407-14-370

75. Miyashita T, Miki K, Kamigaki T, Makino I, Tajima H, Nakanuma S, et al. Low-dose valproic acid with low-dose gemcitabine augments MHC class I-related chain A/B expression without inducing the release of soluble MHC class I-related chain A/B. Oncol Lett. (2017) 14:5918-26. doi: $10.3892 / \mathrm{ol} .2017 .6943$

76. Hu XT, Zhu BL, Zhao LG, Wang JW, Liu L, Lai YJ, et al. Histone deacetylase inhibitor apicidin increases expression of the alpha-secretase ADAM10 through transcription factor USF1-mediated mechanisms. FASEB J. (2017) 31:1482-93. doi: 10.1096/fj.201600961RR

77. Shi X, Li M, Cui M, Niu C, Xu J, Zhou L, et al. Epigenetic suppression of the antitumor cytotoxicity of NK cells by histone deacetylase inhibitor valproic acid. Am J Cancer Res. (2016) 6:600-14.

78. Ni L, Wang L, Yao C, Ni Z, Liu F, Gong C, et al. The histone deacetylase inhibitor valproic acid inhibits NKG2D expression in natural killer cells through suppression of STAT3 and HDAC3. Sci Rep. (2017) 7:45266. doi: $10.1038 /$ srep45266

79. Shiraishi K, Mimura K, Kua LF, Koh V, Siang LK, Nakajima S, et al. Inhibition of MMP activity can restore NKG2D ligand expression in gastric cancer, leading to improved NK cell susceptibility. J Gastroenterol. (2016) 51:1101-11. doi: 10.1007/s00535-016-1197-x

80. Zocchi MR, Camodeca C, Nuti E, Rossello A, Vene R, Tosetti F, et al. ADAM10 new selective inhibitors reduce NKG2D ligand release sensitizing Hodgkin lymphoma cells to NKG2D-mediated killing. Oncoimmunology (2015) 5:e1123367. doi: 10.1080/2162402X.2015.1123367

81. Cathcart J, Pulkoski-Gross A, Cao J. Targeting matrix metalloproteinases in cancer: bringing new life to old ideas. Genes Dis. (2015) 2:26-34. doi: 10.1016/j.gendis.2014.12.002

82. Kohga K, Takehara T, Tatsumi T, Ishida H, Miyagi T, Hosui A, et al. Sorafenib inhibits the shedding of major histocompatibility complex class I-related chain A on hepatocellular carcinoma cells by down-regulating a disintegrin and metalloproteinase 9. Hepatology (2010) 51:1264-73. doi: 10.1002/hep. 23456

83. Black RA, Rauch CT, Kozlosky CJ, Peschon JJ, Slack JL, Wolfson $\mathrm{MF}$, et al. A metalloproteinase disintegrin that releases tumour-necrosis factor-alpha from cells. Nature (1997) 385:729-33. doi: 10.1038/38 $5729 \mathrm{a} 0$

84. Garton KJ, Gough PJ, Blobel CP, Murphy G, Greaves DR, Dempsey PJ, et al. Tumor necrosis factor-alpha-converting enzyme (ADAM17) mediates the cleavage and shedding of fractalkine (CX3CL1). J Biol Chem. (2001) 276:37993-8001. doi: 10.1074/jbc.M106434200

85. Ferrari de Andrade L, Tay RE, Pan D, Luoma AM, Ito Y, Badrinath $\mathrm{S}$, et al. Antibody-mediated inhibition of MICA and MICB shedding promotes NK cell-driven tumor immunity. Science (2018) 359:1537-42. doi: 10.1126/science.aao0505

86. Wu J. Antibody targeting soluble NKG2D ligand sMIC refuels and invigorates the endogenous immune system to fight cancer. Oncoimmunology (2015) 5:e1095434. doi: 10.1080/2162402X.2015.10 95434

87. Weil S, Memmer S, Lechner A, Huppert V, Giannattasio A, Becker T, et al. Natural killer group 2D ligand depletion reconstitutes natural killer cell immunosurveillance of head and neck squamous cell carcinoma. Front Immunol. (2017) 8:387. doi: 10.3389/fimmu.2017.00387

88. Raab S, Steinbacher J, Schmiedel BJ, Kousis PC, Steinle A, Jung G, et al. Fc-optimized NKG2D-Fc constructs induce NK cell antibodydependent cellular cytotoxicity against breast cancer cells independently of HER2/neu expression status. J Immunol. (2014) 193:4261-72. doi: 10.4049/jimmunol.1400872

89. Steinbacher J, Baltz-Ghahremanpour K, Schmiedel BJ, Steinle A, Jung G, Kubler A, et al. An Fc-optimized NKG2D-immunoglobulin $\mathrm{G}$ fusion protein for induction of natural killer cell reactivity against leukemia. Int $J$ Cancer (2015) 136:1073-84. doi: 10.1002/ijc. 29083

90. Zhang T, Sentman CL. Cancer immunotherapy using a bispecific NK receptor fusion protein that engages both $\mathrm{T}$ cells and tumor cells. Cancer Res (2011) 71:2066-76. doi: 10.1158/0008-5472.CAN-10-3200

91. Godbersen C, Coupet TA, Huehls AM, Zhang T, Battles MB, Fisher JL, et al. NKG2D ligand-targeted bispecific T-Cell engagers lead to robust antitumor activity against diverse human tumors. Mol Cancer Ther. (2017) 16:1335-46. doi: 10.1158/1535-7163.MCT-16-0846

92. Xia Y, Chen B, Shao X, Xiao W, Qian L, Ding Y, et al. Treatment with a fusion protein of the extracellular domains of NKG2D to IL-15 retards colon cancer growth in mice. J Immunother. (2014) 37:257-66. doi: 10.1097/CJI.0000000000000033

93. Tan L, Han S, Ding S, Xiao W, Ding Y, Qian L, et al. Chitosan nanoparticlebased delivery of fused NKG2D-IL-21 gene suppresses colon cancer growth in mice. Int J Nanomedicine (2017) 12:3095-107. doi: 10.2147/IJN.S1 28032

94. Chen Y, Chen B, Yang T, Xiao W, Qian L, Ding Y, et al. Human fused NKG2D-IL-15 protein controls xenografted human gastric cancer through the recruitment and activation of NK cells. Cell Mol Immunol. (2017) 14:293-307. doi: 10.1038/cmi.2015.81

95. Horowitz MM, Gale RP, Sondel PM, Goldman JM, Kersey J, Kolb HJ, et al. Graft-versus-leukemia reactions after bone marrow transplantation. Blood (1990) 75:555-62.

96. Estey E, Dohner H. Acute myeloid leukaemia. Lancet (2006) 368:1894-907. doi: 10.1016/S0140-6736(06)69780-8

97. Carapito R, Aouadi I, Ilias W, Bahram S. Natural killer group 2, Member D/NKG2D ligands in hematopoietic cell transplantation. Front Immunol. (2017) 8:368. doi: 10.3389/fimmu.2017. 00368

98. Hofer E, Koehl U. Natural killer cell-based cancer immunotherapies: from immune evasion to promising targeted cellular therapies. Front Immunol. (2017) 8:745. doi: 10.3389/fimmu.2017.00745

99. Kloess S, Huenecke S, Piechulek D, Esser R, Koch J, Brehm C, et al. IL-2-activated haploidentical NK cells restore NKG2D-mediated NKcell cytotoxicity in neuroblastoma patients by scavenging of plasma MICA. Eur J Immunol. (2010) 40:3255-67. doi: 10.1002/eji.2010 40568

100. Sentman CL, Meehan KR. NKG2D CARs as cell therapy for cancer. Cancer J. (2014) 20:156-9. doi: 10.1097/PPO.0000000000000029

101. Zhang $\mathrm{T}$, Lemoi BA, Sentman CL. Chimeric NK-receptor-bearing $\mathrm{T}$ cells mediate antitumor immunotherapy. Blood (2005) 106:1544-51. doi: 10.1182/blood-2004-11-4365

102. Zhang T, Barber A, Sentman CL. Generation of antitumor responses by genetic modification of primary human $\mathrm{T}$ cells with a chimeric NKG2D receptor. Cancer Res. (2006) 66:5927-33. doi: 10.1158/0008-5472.CAN-06-0130

103. Barber A, Zhang T, DeMars LR, Conejo-Garcia J, Roby KF, Sentman CL. Chimeric NKG2D receptor-bearing $\mathrm{T}$ cells as immunotherapy for ovarian cancer. Cancer Res. (2007) 67:5003-8. doi: 10.1158/0008-5472. CAN-06-4047

104. Song DG, Ye Q, Santoro S, Fang C, Best A, Powell DJ Jr. Chimeric NKG2D CAR-expressing $\mathrm{T}$ cell-mediated attack of human ovarian cancer is enhanced by histone deacetylase inhibition. Hum Gene Ther. (2013) 24:295-305. doi: 10.1089/hum.2012.143

105. VanSeggelen H, Hammill JA, Dvorkin-Gheva A, Tantalo DG, Kwiecien JM, Denisova GF, et al. T cells engineered with chimeric antigen receptors targeting NKG2D ligands display lethal toxicity in mice. Mol Ther. (2015) 23:1600-10. doi: 10.1038/mt.2015.119

106. Sentman ML, Murad JM, Cook WJ, Wu MR, Reder J, Baumeister SH, et al. Mechanisms of Acute Toxicity in NKG2D Chimeric Antigen Receptor T Cell-Treated Mice. J Immunol. (2016) 197:4674-85. doi: 10.4049/jimmunol.1600769 
107. Balint S, Lopes FB, Davis DM. A nanoscale reorganization of the IL-15 receptor is triggered by NKG2D in a ligand-dependent manner. Sci Signal. (2018) 11:eaal3606. doi: 10.1126/scisignal. aal3606

108. Molfetta R, Quatrini L, Capuano C, Gasparrini F, Zitti B, Zingoni A, et al. c-Cbl regulates MICA- but not ULBP2-induced NKG2D downmodulation in human NK cells. Eur J Immunol. (2014) 44:2761-70. doi: 10.1002/eji.201444512

109. Schmiedel D, Mandelboim O. Disarming cellular alarm systemsmanipulation of stress-induced NKG2D ligands by human herpesviruses. Front Immunol. (2017) 8:390. doi: 10.3389/fimmu.2017.00390
Conflict of Interest Statement: The authors declare that the research was conducted in the absence of any commercial or financial relationships that could be construed as a potential conflict of interest.

Copyright (c) 2018 Schmiedel and Mandelboim. This is an open-access article distributed under the terms of the Creative Commons Attribution License (CC BY). The use, distribution or reproduction in other forums is permitted, provided the original author(s) and the copyright owner(s) are credited and that the original publication in this journal is cited, in accordance with accepted academic practice. No use, distribution or reproduction is permitted which does not comply with these terms. 